\title{
Efecto de termosonicación y pasteurización sobre propiedades fisicoquímicas, microbiológicas y nutracéuticas en bebidas de maíz
}

\author{
Effect of thermosonication and pasteurization on physicochemical, microbiological \\ and nutraceutical properties in maize beverages
}

\begin{abstract}
Pablo Alan Rodríguez-Salinas', Vania Urías-Orona², Dolores Muy-Rangel ${ }^{3}$, José Basilio-Heredia ${ }^{3}$, Angela SuarezJacobo ${ }^{4}$, Juan Gabriel Báez-González ${ }^{5}$, Francisco Zavala-García', Guillermo Niño-Medina ${ }^{1 *}$

* Universidad Autónoma de Nuevo León, Facultad de Agronomía. Francisco Villa S/N, Col. Ex Hacienda El Canadá, C.P. 66050. General Escobedo, Nuevo León, México.

2 Universidad Autónoma de Nuevo León, Facultad de Salud publica y Nutrición. Av. Dr. Eduardo Aguirre Pequeño y Yuridia, Col. Mitras Centro, C.P. 64460. Monterrey, Nuevo León, México.

Centro de Investigación en Alimentación y Desarrollo (CIAD), A.C., Coordinación Culiacán. Carretera Culiacán a El Dorado km 5.5, C.P. 80110. Col. Campo el Diez, Culiacán, Sinaloa, México.

${ }^{4}$ Centro de Investigación y Asistencia en Tecnología y Diseño del Estado de Jalisco (CIATEJ), A.C., Unidad Noreste. Parque de Investigación e Innovación Tecnológica (PIIT), C.P. 66629. Apodaca, Nuevo León, México.

Universidad Autónoma de Nuevo León, Facultad de Ciencias Biológicas. Av. Universidad S/N, C.P. 66451 San Nicolás de los Garza, Nuevo León, México.
\end{abstract}

\section{RESUMEN}

El maíz es utilizado como una de las principales fuentes en la nutrición humana. Gracias a su aporte nutrimental se utiliza en la preparación de bebidas artesanales o tradicionales. Debido a que existe poca información sobre formulación de bebidas y su comportamiento frente a tratamientos emergentes o tradicionales de conservación, el objetivo de este trabajo fue evaluar el efecto de un tratamiento de conservación con termosonicación y un tratamiento tradicional como la pasteurización sobre color, calidad microbiológica, parámetros fisicoquímicos y propiedades nutracéuticas de bebidas de maíz blanco y morado. Los resultados en las bebidas mostraron modificaciones de color y parámetros fisicoquímicos ( $\mathrm{pH}$, acidez titulable y sólidos totales) y contenido de microorganismos. No se observaron diferencias en los compuestos fenólicos (95.23-128.29 mg EAG/L), flavonoides (43.41-72.30 mg EC/L) y antocianinas (0.89-1.20 mg EC3G/L). La bebida de maíz blanco presentó mayor contenido de ácido ferúlico (45.77-48.90 mg/L), mientras que la de maíz morado presentó mayores valores de ácido clorogénico (37.14-39.58 $\mathrm{mg} / \mathrm{L})$, cafeico (33.26 mg/L) y capacidad antioxidante por DPPH (311.48-384.78 $\mu \mathrm{mol}$ ET/L) y FRAP (1093.33-1566.67 $\mu \mathrm{mol}$ ET/L). En conclusión, la pasteurización en bebidas de maíz favoreció el incremento en la capacidad antioxidante y una mayor eficiencia en la calidad microbiológica.

Palabras clave: Calidad microbiológica, compuestos fenólicos, termosonicación, pasteurización, capacidad antioxidante.

\section{ABSTRACT}

Maize is one of the main sources in human nutrition. Thanks to its nutritional contribution, it is used in the crafted or traditional beverages preparation. Since there is little information on the beverage formulation and their behavior in emerging or traditional preservation treatments, the

Volumen XXIII, Número 1 objective of this work was to evaluate the effect of thermosonication as a preservation treatment, and a pasteurization traditional treatment, on color, microbiological quality, physicochemical parameters and nutraceutical properties of white and purple maize beverages. The results show changes in color and physicochemical parameters $(\mathrm{pH}$, titratable acidity and total solids) and content of microorganisms. No differences were observed in phenolic compounds (95.23-128.29 mg EAG/L), flavonoids (43.41-72.30 mg EC/L) and anthocyanins (0.89-1.20 mg EC3G/L). The white maize beverage had a higher ferulic acid content (45.77-48.90 mg / L), while the purple maize showed higher chlorogenic acid values (37.14$39.58 \mathrm{mg} / \mathrm{L})$, caffeic $(33.26 \mathrm{mg} / \mathrm{L})$ and antioxidant capacity of DPPH (311.48-384.78 $\mu \mathrm{mol}$ ET/L) and FRAP (1093.33-1566.67 $\mu \mathrm{mol} E T / L)$. In conclusion, pasteurization in maize beverages favored the increase of the antioxidant capacity and a greater efficiency in microbiological quality.

Keywords: Microbiological quality, phenolic compounds, thermosonication, pasteurization, antioxidant capacity.

\section{INTRODUCCIÓN}

El maíz (Zea mays L.) es utilizado como una fuente de alimentación en el mundo (Ranum et al., 2014). Los cereales como el maíz poseen alto contenido de compuestos fenólicos. Estos compuestos pueden encontrarse de forma libre o ligada, un ejemplo de esto es el ácido ferúlico, que en maíz existe principalmente de forma ligada, y se encuentra formando parte de la estructura de la pared celular del pericarpio y brinda una barrera física y química de protección contra la oxidación, patógenos, ataque de insectos y animales (Wang et al., 2014; Boz, 2015). Además del ácido ferúlico del pericarpio, existen algunas variedades de maíz que poseen pigmentación en el grano, la cual, también puede estar presente en el pericarpio y/o en la capa de aleurona, con variación en las tonalidades rojas, azules, moradas y ne-

*Autor para correspondencia: Guillermo Niño Medina Correo electrónico: guillermo.ninomd@uanl.edu.mx

Recibido: 29 de enero de 2020 Aceptado: $\mathbf{2 1}$ de septiembre de 2020 
gras. La pigmentación del grano se atribuye a la presencia de compuestos antioxidantes como antocianinas, flavonoides y taninos condensados, todos ellos con potencial para producir alimentos o bebidas nutraceúticas (Akonor et al., 2014; Chaves-López et al., 2014; Urias-Lugo et al., 2015).

Los productos elaborados a partir del grano pigmentado de maíz son considerados como antioxidantes (UriasLugo et al., 2015) y en algunos países son preparadas bebidas a partir de granos de maíz pigmentados (Akonor et al., 2014; Chaves-López et al., 2014). Estas bebidas gracias a su aporte nutrimental son aceptadas, sin embargo, sólo se elaboran de una forma artesanal, tradicional o regional utilizando el tipo de maíz disponible en el lugar en donde se elabore, debido a esto, existe poca información respecto formulaciones, o el efecto en la calidad de estas frente a procesos de conservación (Wang et al., 2014; Carrera et al., 2015; Rodríguez-Salinas et al., 2019).

En la conservación de alimentos, los tratamientos térmicos son los más utilizados debido a que brinda un producto seguro para el consumo humano y una mayor efectividad en la destrucción de microorganismos causantes del deterioro de los alimentos (Wang et al., 2014). Estos tratamientos térmicos además de ser una alternativa económica para generar la inocuidad de los alimentos pueden provocar cambios indeseables, como fisicoquímicos, pérdida de nutrientes, formación de componentes por reacción térmica y perdida de apariencia (Rico et al., 2007; Suarez-Jacobo et al., 2010), así como perdida de compuestos con capacidad antioxidante (Abdullah y Chin, 2014). En alimentos líquidos el tratamiento térmico más utilizado para la conservación es la pasteurización (Suarez-Jacobo et al., 2011). El efecto de la pasteurización sobre la destrucción de microorganismos e inactivación de enzimas que pueden afectar la apariencia del producto depende en gran medida de la temperatura (Abdullah y Chin, 2014), por otro lado, los cambios generados durante el tiempo de procesamiento, por lo que muchos estudios se han enfocado en el estudio de altas temperaturas con cortos tiempos, utilizando equipos industriales de pasteurización (Rico et al., 2007; Estrada et al., 2010). Sin embargo, es reportado que algunos procesos de pasteurización eficientes utilizan temperaturas de van de los 80,90 a $95^{\circ} \mathrm{C}$ por $10 \mathrm{~min}$ aproximadamente (Rawson et al., 2011), pero según Estrada et al. (2010) $85^{\circ} \mathrm{C}$ por 5 min puede considerarse como una pasteurización artesanal.

Por otro lado, y debido a los inconvenientes causados por el uso de temperaturas superiores a los $80^{\circ} \mathrm{C}$ que puede provocar cambios físicos, químicos y biológicos indeseables en los alimentos líquidos el uso la tecnologías emergentes como la termosonicación, la cual combina calentamiento moderado con temperaturas que van de los 37 a $75^{\circ} \mathrm{C}$ con el uso del ultrasonido con frecuencias de los $20 \mathrm{kHz}$ a 10 $\mathrm{MHz}$, representa una técnica alternativa para evitar cambios indeseables y favorecer la inactivación de enzimas y microorganismos causales del deterioro de este tipo de alimentos. La acción de la termosonicación provoca el proceso de cavitación, el cual genera burbujas de vapor por los cambios de presión que después explotan generando calor y presión, dando como resultado una esterilización localizada y disrupción celular (Abdullah y Chin, 2014; Anaya-Esparza et al 2016; Jiménez-Sánchez et al., 2017).

Debido a lo anterior, los cambios generados en los alimentos por los tratamientos térmicos y que hoy en día los consumidores demandan productos con las cualidades de un alimento fresco (Jiménez-Sánchez et al., 2017), el objetivo de este trabajo fue evaluar dos bebidas de maíz, una de maíz blanco y una de maíz morado, así como el efecto de un tratamiento de conservación de tecnología emergente como la termosonicación y un tratamiento térmico tradicional como la pasteurización a $85^{\circ} \mathrm{C}$ por $5 \mathrm{~min}$, sobre color contenido de microorganismos, parámetros fisicoquímicos y propiedades nutracéuticas.

\section{MATERIALES Y MÉTODOS \\ Preparación y obtención de harina}

La bebida de maíz se formuló y preparó con el grano de maíz blanco y morado obtenido de la Facultad de Agronomía de la Universidad Autónoma de Nuevo León. La harina de maíz se obtuvo tostando $500 \mathrm{~g}$ de grano en un comal de acero a $145 \pm 5^{\circ} \mathrm{C}$ por $30 \mathrm{~min}$, la temperatura se monitoreo cada 5 min con un termómetro de infrarrojo; después el grano tostado se molió y tamizó hasta obtener un tamaño de partícula de $0.59 \mathrm{~mm}$ (malla 30).

\section{Formulación de bebida de maíz}

Los ingredientes de las bebidas de maíz en base al porcentaje consistieron en agua purificada $94.83 \%$, harina de maíz (Zea mays L.), blanco o morado, según el caso $2.37 \%$, azúcar $1.90 \%$, extracto natural de Vainilla (Vanilla planifolia Jacks). $0.47 \%$ de canela (Cinnamomun verum J. Presl) $0.14 \%$, de Stevia (Stevia rebaudiana Bert.) $0.14 \%$, goma xantana 0.09 $\%$, carboximetilcelulosa (CMC) $0.05 \%$.

\section{Tratamientos}

Las bebidas de maíz blanco y morado recién preparadas fueron separadas por lotes de $500 \mathrm{~mL}$ y refrigeradas toda la noche a $4^{\circ} \mathrm{C}$. Después se llevaron a cabo dos tratamientos de conservación; el tratamiento de termosonicación se realizó en un procesador ultrasónico UIP500hdt (Hielscher USA, Inc., Ringwood, USA) (20 kHz y $500 \mathrm{~W}$ ) a $80 \%$ de amplitud, con un senotrodo de $18 \mathrm{~mm}$ con control de temperatura a $45^{\circ} \mathrm{C}$ por $20 \mathrm{~min}$, y el tratamiento térmico de pasteurización a $85^{\circ} \mathrm{C}$ por $5 \mathrm{~min}$. La bebida sin proceso de conservación fue utilizada como control.

\section{Parámetros cromáticos}

El color de la bebida se determinó llenando una cubeta espectrofotométrica de $3 \mathrm{~mL}$ y las mediciones del color se realizaron con un colorímetro (CR-20, Konica Minolta, Japan) con iluminante D65 y un ángulo de $10^{\circ}$. Los parámetros cromáticos se basaron en escala CIELAB $\left(L^{*} a^{*} b^{*}\right)$ y CIELCH $\left(L^{*}\right.$ $\left.C^{*} h\right)$. 


\section{Calidad microbiológica}

La calidad microbiológica se determinó en base a lo reportado por Rodríguez-Salinas et al. (2019) con diluciones en agua peptonada, para conteos decimales. Los mesófilos aerobios, lactobacilos, enterobacterias, hongos y levaduras, y esporulación fueron enumerados en agar PCA (Plate Count Agar, $37^{\circ} \mathrm{C} \times 48 \mathrm{~h}$ ), MRS (Man-Rogosa-Sharpe, $37^{\circ} \mathrm{C} \times 48 \mathrm{~h}$ con $5 \%$ de $\mathrm{CO}_{2}$ ), VRBG (Violet Red Bile Glucose, $37^{\circ} \mathrm{C} \times 24 \mathrm{~h}$ ), PDA (Potato Dextrose Agar, $25^{\circ} \mathrm{C} \times 5$ días) acidificado y PCA (Plate Count Agar $37^{\circ} \mathrm{C} \times 48 \mathrm{~h}$ ), respectivamente. Los resultados se expresaron en logaritmo de unidades formadoras de colonias por mililitro de muestra (log ufc/mL).

\section{Parámetros fisicoquímicos}

El pH se registró con un potenciómetro (Corning, 440), los sólidos totales, en un refractómetro de mano (Master-M 2313, ATAGO, Japan), y acidez titulable en base a lo descrito por Akonor et al. (2014).

\section{Compuestos fenólicos}

Los fenoles totales, flavonoides totales y taninos condensados se realizaron con base a lo reportado por LópezContreras et al. (2015). Los fenoles totales se cuantificaron usando una curva de calibración con ácido gálico (0-200 $\mathrm{mg} / \mathrm{L}$ ) y los resultados se expresaron en miligramos equivalentes de ácido gálico por litro (mg EAG/L). Los flavonoides totales y taninos condensados se cuantificaron usando curvas de calibración con catequina $(0-200 \mathrm{mg} / \mathrm{L})$ y los resultados se expresaron en miligramos equivalentes de catequina por litro (mg EC/L). Las antocianinas totales se extrajeron y cuantificaron en base a lo descrito por Rodríguez-Salinas et al, (2019). Los resultados se expresaron en miligramos equivalentes de cianidina-3-glucósido por litro muestra ( $\mathrm{mg}$ ECG/L).

\section{Extracción de compuestos fenólicos y análisis cromato- gráfico (HPLC)}

Debido a la viscosidad de la muestra se realizó una extracción siguiendo la metodología descrita por NiñoMedina et al. (2017), después, los extractos se filtraron con un acrodisco de $0.25 \mathrm{~mm}$ con un tamaño de poro de $45 \mu \mathrm{m}$ y se almacenaron protegidos de la luz hasta su posterior análisis.

\section{Análisis cromatográfico (HPLC)}

Los análisis se realizaron en un equipo HPLC Agilent Technologies 1260 Infinity (Agilent, Santa Clara, CA, USA), bomba cuaternaria Agilent 1260 (G1311B) con auto-muestreador, un detector con arreglo de diodos (DAD) Agilent 1260 (G4212B) y una columna analítica ZORBAX Eclipse Plus C-18 (100 mm x $3 \mathrm{~mm}$ de i.d., $5 \mu \mathrm{m}$ ). Las fases móviles fueron agua $\left(0.1 \%\right.$ de $\left.\mathrm{CH}_{3} \mathrm{COOH}, \mathrm{A}\right)$ y $\mathrm{MeOH}\left(0.1 \%\right.$ de $\left.\mathrm{CH}_{3} \mathrm{COOH}, \mathrm{B}\right)$, con flujo de $0.6 \mathrm{~mL} / \mathrm{min}$ a $25^{\circ} \mathrm{C}$, con un tiempo de corrida de 60 min e inyección de muestra de $10 \mu \mathrm{L}$. El gradiente utilizado fue el reportado por Santos et al. (2014), y se obtuvo el espectro de los compuestos en la región UV (200-400 nm). Los compuestos fenólicos individuales se cuantificaron usando una curva de calibración con ácido gálico, catequina, ácido clorogénico, ácido cafeico, ácido cumárico, ácido ferúlico, ácido sinápico y quercetina (0-200 mg/L) y los resultados se expresaron en miligramos equivalentes de cada compuesto identificado por litro de muestra ( $\mathrm{mg} / \mathrm{L}$ ).

\section{Capacidad antioxidante}

La capacidad antioxidante DPPH, ABTS y FRAP se realizó mediante la metodología de López-Contreras et al. (2015), los niveles fueron calculados con una curva de calibración con Trolox (0-500 $\mu \mathrm{mol} / \mathrm{L})$ y los resultados se expresaron en micromoles equivalentes de Trolox por litro ( $\mu \mathrm{mol} E T / L)$.

\section{Viscosidad}

Las curvas de flujo y viscosidad se obtuvieron mediante una prueba rotacional con un reómetro Anton Paar (Rheolab QC, Austria) en función de la tasa de corte de 1 a $500 \mathrm{~s} / 1$, utilizando una geometría CC27 a una temperatura de $25^{\circ} \mathrm{C}$.

\section{Análisis estadístico}

Los resultados de las muestras se sometieron a un análisis de varianza (ANOVA) de un factor y las medias se sometieron a la prueba de Tukey $(P \leq 0.05)$ analizadas mediante el paquete estadístico Minitab (Versión 14). De todas las determinaciones se realizaron cinco repeticiones y los datos se expresan como media \pm desviación estándar.

\section{RESULTADOS Y DISCUSIÓN}

\section{Parámetros cromáticos}

Los valores de luminosidad $\left(L^{*}\right)$ entre las bebidas de maíz blanco y morado mostraron diferencias significativas con valores de 35.20 a 37.54 y 31.20 a 36.46 , respectivamente, con los valores más altos en la bebida de maíz blanco (Tabla 1). También se encontraron diferencias entre los tratamientos de cada tipo de bebida, obteniendo los menores valores de $L^{*}$ en el tratamiento de pasteurización y los mayores en termosonicación.

Estos resultados en las bebidas de maíz blanco y morado indican que el tratamiento de termosonicación no afectó la $L^{*}$ de las bebidas (Abdullah y Chin, 2014). Sin embargo, el tratamiento de pasteurización ocasionó oscurecimiento de la bebida, lo que se puede atribuir a la reacción entre azúcares reductores y grupos amino, conocida como reacción de Maillard, reacción frecuente en el procesamiento o cocción de los alimentos (Abdullah y Chin, 2014; Tamanna y Mahmood, 2015; Rodríguez-Salinas et al., 2019).

Los valores de $a^{*}$ (Tabla 1 ) entre las bebidas de maíz blanco y morado variaron de 1.98 a 2.74 y 2.00 a 2.84 , respectivamente, sin diferencia entre el tipo de bebida. Con respecto a los tratamientos de cada tipo de bebida, en termosonicación se observaron los valores más bajos en $a^{*}$ en ambas bebidas, mientras que en la pasteurización, sólo en la bebida de maíz blanco se observó el incremento de los valores de $a^{*}$, con diferencias estadísticas entre tratamientos de cada tipo de bebida. El valor $b^{*}$ de las bebidas de maíz blanco y morado mostraron diferencia estadística entre tipo bebida con 
Tabla 1. Parámetros cromáticos de bebidas de maíz blanco y maíz morado.

Table 1. Chromatic parameter of white and purple maize beverages.

\begin{tabular}{lcccccc}
\hline \multirow{2}{*}{ Parámetro } & \multicolumn{3}{c}{ Bebida de Maíz Blanco } & \multicolumn{3}{c}{ Bebida de maíz Morado } \\
& Control & Termosonicación & Pasteurización & Control & Termosonicación & Pasteurización \\
\hline$L^{*}$ & $36.26 \pm 0.47^{\mathrm{ab}}$ & $37.54 \pm 0.86^{\mathrm{a}}$ & $35.2 \pm 1.32^{\mathrm{b}}$ & $33.92 \pm 0.13^{\mathrm{b}}$ & $36.46 \pm 0.17^{\mathrm{a}}$ & $31.2 \pm 0.19^{\mathrm{c}}$ \\
\hline$a^{*}$ & $2.5 \pm 0.12^{\mathrm{b}}$ & $1.98 \pm 0.15^{\mathrm{c}}$ & $2.74 \pm 0.05^{\mathrm{a}}$ & $2.84 \pm 0.05^{\mathrm{a}}$ & $2.00 \pm 0.07^{\mathrm{c}}$ & $2.66 \pm 0.09^{\mathrm{b}}$ \\
\hline$b^{*}$ & $11.04 \pm 0.52^{\mathrm{b}}$ & $13.34 \pm 0.80^{\mathrm{a}}$ & $10.80 \pm 0.45^{\mathrm{b}}$ & $9.00 \pm 0.07^{\mathrm{b}}$ & $11.18 \pm 0.37^{\mathrm{a}}$ & $7.32 \pm 0.08^{\mathrm{c}}$ \\
\hline$C^{*}$ & $11.32 \pm 0.56^{\mathrm{b}}$ & $13.48 \pm 0.83^{\mathrm{a}}$ & $11.04 \pm 0.54^{\mathrm{b}}$ & $9.42 \pm 0.04^{\mathrm{b}}$ & $11.36 \pm 0.33^{\mathrm{a}}$ & $7.80 \pm 0.10^{\mathrm{c}}$ \\
\hline$h$ & $77.2 \pm 0.12^{\mathrm{b}}$ & $81.5 \pm 0.19^{\mathrm{a}}$ & $75.62 \pm 0.46^{\mathrm{c}}$ & $72.44 \pm 0.21^{\mathrm{b}}$ & $79.76 \pm 0.34^{\mathrm{a}}$ & $70.10 \pm 0.41^{\mathrm{c}}$ \\
\hline Color visual & & & & & & \\
\hline
\end{tabular}

Diferente letra en la misma línea dentro de cada tipo de bebida de maíz indica diferencia estadística significativa $(P \leq 0.05)$. Media \pm Desviación estándar $(n=5)$.

Different letter in same line of each kind of maize beverages indicate significant statistical differences $(P \leq 0.05)$. Means \pm Standard deviation $(n=5)$.

valores de 10.80 a 13.34 y 7.32 a 11.18 , respectivamente, con los mayores valores en la bebida de maíz blanco (Tabla 1). Los tratamientos de cada tipo de bebida mostraron diferencias estadísticas, en $b^{*}$ los menores valores se encontraron en la pasteurización y los mayores en termosonicación.

Los resultados en los valores de $a^{*}$ y $b^{*}$ en las bebidas mostraron que el tratamiento de termosonicación incrementó el color verde y amarillo, respectivamente. Esto se debe a que la termosonicación promovió la homogenización de los ingredientes de la bebida y mejoró el color con la distribución de pigmentos naturales (Abdullah y Chin, 2014; Jiménez-Sánchez et al., 2017). Con respecto a la pasteurización los valores de $a^{*}$ y $b^{*}$ incrementaron el color rojo y azul, respectivamente. Estos cambios demuestran el efecto de los tratamientos térmicos sobre el color, probablemente debido a la aceleración de productos de la reacción de Maillard (Jiménez-Sánchez et al., 2017; Mondaca-Navarro et al., 2017; Rodríguez-Salinas et al., 2019).

Los valores de Cromaticidad $\left(C^{*}\right)$ y ángulo Hue $(h)$ (Tabla 1), en las bebidas de maíz blanco y morado mostraron diferencias estadísticas en el tipo de bebida con valores de $C^{*}$ de 11.04 a 13.48 y 7.80 a 11.36 , respectivamente, mientras que en $h$ de 75.62 a 81.5 y 70.10 a 76.76 , respectivamente, con los mayores valores en la bebida de maíz blanco. Los tratamientos aplicados a cada tipo de bebida mostraron diferencias estadísticas. En ambas bebidas se observó que los valores más bajos correspondieron a la pasteurización y los mayores a la termosonicación.

Los valores de $C^{*}$ y $h$ en las bebidas disminuyeron con el tratamiento de pasteurización, a diferencia del tratamiento con termosonicación, el cual incremento los valores de estos parámetros. Los parámetros de $C^{*}$ y $h$ son más apropiados para la interpretación del color, ya que $C^{*}$ indica la saturación del color, el cual en las muestras de este estudio se encontraron en la zona del color gris. En cuanto al valor $h$, este expresa el color percibido por el ojo humano, para este estudio los valores $h$ de las muestras se expresaron en color amarillo (López-Contreras et al., 2015).

\section{Calidad microbiológica}

El contenido de microorganismos (Tabla 2) en los tratamientos control de las bebidas de maíz blanco y morado mostraron valores para mesófilos aerobios de 6.96 y $7.31 \mathrm{log}$ $\mathrm{ufc} / \mathrm{mL}$, en coliformes de 5.45 y $7.06 \mathrm{log}$ ufc $/ \mathrm{mL}$, con mayores valores en la bebida de maíz morado, y lactobacilos 1.61 y $1.58 \mathrm{log}$ ufc $/ \mathrm{mL}$, respectivamente. En los tratamientos de las bebidas de maíz blanco y morado, en termosonicación se observó disminución de mesófilos aerobios con valores de 4.37 y $4.64 \log \mathrm{ufc} / \mathrm{mL}$, coliformes 3.06 y 3.88 y lactobacilos de 1.76 y 1.58 , respectivamente, mientras que en el tratamiento de pasteurización de ambas bebidas, los microorganismos no fueron detectados.

Tabla 2. Calidad microbiológica de bebidas de maíz blanco y maíz morado.Table 2. Microbiological quality of white and purple maize beverages.

\begin{tabular}{|c|c|c|c|c|c|c|}
\hline \multirow{2}{*}{ Análisis } & \multicolumn{3}{|c|}{ Bebida de Maíz Blanco } & \multicolumn{3}{|c|}{ Bebida de Maíz Morado } \\
\hline & Control & Termosonicación & Pasteurización & Control & Termosonicación & Pasteurización \\
\hline Mesófilos aerobios & $6.96 \pm 0.05^{c}$ & $4.37 \pm 0.03^{b}$ & $\mathrm{ND}^{\mathrm{a}}$ & $7.31 \pm 0.04^{c}$ & $4.64 \pm 0.02^{b}$ & $\mathrm{ND}^{\mathrm{a}}$ \\
\hline Enterobacterias & $5.45 \pm 0.03^{c}$ & $3.06 \pm 0.02^{b}$ & $\mathrm{ND}^{\mathrm{a}}$ & $7.06 \pm 0.04^{c}$ & $3.88 \pm 0.01^{b}$ & $\mathrm{ND}^{\mathrm{a}}$ \\
\hline Lactobacilos & $1.61 \pm 0.02^{\mathrm{b}}$ & $1.76 \pm 0.02^{c}$ & $\mathrm{ND}^{\mathrm{a}}$ & $1.58 \pm 0.02^{b}$ & $1.58 \pm 0.02^{\mathrm{b}}$ & $\mathrm{ND}^{\mathrm{a}}$ \\
\hline Hongos y levadura & $\mathrm{ND}^{\mathrm{a}}$ & $N^{a}$ & $\mathrm{ND}^{\mathrm{a}}$ & $N^{a}$ & $\mathrm{ND}^{\mathrm{a}}$ & $\mathrm{ND}^{\mathrm{a}}$ \\
\hline Esporas & $N^{a}$ & $\mathrm{ND}^{\mathrm{a}}$ & $N D^{a}$ & $N^{a}$ & $N D^{a}$ & $\mathrm{ND}^{\mathrm{a}}$ \\
\hline
\end{tabular}

Diferente letra en la misma línea dentro de cada tipo bebida de maíz indica diferencia estadística significativa $(P \leq 0.05)$. Media \pm Desviación estándar $(n=5)$. ND = No detectado.

Different letters in same line of each kind of maize beverages indicate significant statistical differences $(P \leq 0.05)$. Means \pm Standard deviation. $(n=5) . N D=$ Not detected. 
La carga microbiana de las bebidas control de maíz blanco y morado después del tratamiento de termosonicación mostraron disminución en mesófilos aerobios de 2.59 y 2.67 , en coliformes de 2.39 y 3.18 , sin embargo, a pesar de la reducción de microorganismos, estos resultados son menores a $5 \mathrm{log}$ ufc/mL requeridas por la FDA en el uso de tratamientos con tecnologías emergentes para asegurar la inocuidad de los alimentos (Roig-Sagués et al., 2015). Cabe mencionar que, el efecto de termosonicación está influenciada por la especie del microorganismo y componentes del alimento, ya que cada uno de estos elementos posee resistencia a la fuerza o tensión de rotura, lo que ofrece un efecto en la distribución de la onda generada por el ultrasonido (Abdullah y Chin, 2014; Jiménez-Sánchez et al., 2017), por lo que deben realizarse pruebas para obtener las condiciones óptimas en esta bebida. Por otro lado, la pasteurización provocó la inactivación total de los microorganismos. Estos resultados demuestran que los tratamientos térmicos tradicionales utilizados para la conservación de bebidas como es la pasteurización, resulta ser un proceso eficiente, accesible, seguro y económico para la eliminación de microrganismos causantes del deterioro (Abdullah y Chin, 2014; Aamir et al., 2014).

\section{Parámetros fisicoquímicos}

El pH en la bebida de maíz blanco mostró diferencia con valores de 6.21 a 6.35 con menor valor en el tratamiento control y el mayor en pasteurización (Tabla 3). En la bebida de maíz morado los valores variaron entre 6.23 a 6.29 , sin diferencia entre tratamientos, con el menor valor en la pasteurización y el mayor valor en el control. La acidez titulable no mostró diferencia, debido a que todos los tratamientos mostraron valores de $0.03 \%$ de equivalentes de ácido málico. Los valores en el contenido de sólidos totales de las bebidas de maíz blanco fueron 2.32, 2.35 y 2.41, para el control, termosonicación y pasteurización, respectivamente, con el mayor valor en pasteurización. En cuanto a la bebida de maíz morado los valores fluctuaron de 2.31 a 2.37 con el menor valor para termosonicación y el mayor e igual valor se mostró en el control y pasteurización.

El valor de $\mathrm{pH}$ y contenido de sólidos totales sólo en la bebida de maíz blanco mostró un incremento después del tratamiento de pasteurización, a diferencia de la bebida de maíz morado que obtuvo el comportamiento contrario, sin diferencia estadística entre las muestras. El efecto de la bebida de maíz blanco pudiera ser atribuido a evaporación de agua (Kathiravan et al., 2014), así como la homogenización

Tabla 3. Parámetros fisicoquímicos, compuestos fenólicos y capacidad antioxidantes en bebidas de maíz blanco y maíz morado.

Table 3. Physiochemical parameter, phenolic compounds and antioxidant capacity in white and purple maize beverages.

\begin{tabular}{|c|c|c|c|c|c|c|}
\hline \multirow{2}{*}{ Parámetros } & \multicolumn{3}{|c|}{ Bebida de Maíz Blanco } & \multicolumn{3}{|c|}{ Bebida de maíz Morado } \\
\hline & Control & Termosonicación & Pasteurización & Control & Termosonicación & Pasteurización \\
\hline $\mathrm{pH}$ & $6.21 \pm 0.01^{b}$ & $6.29 \pm 0.10^{\mathrm{ab}}$ & $6.35 \pm 0.01^{\mathrm{a}}$ & $6.29 \pm 0.08^{\mathrm{a}}$ & $6.25 \pm 0.06^{\mathrm{a}}$ & $6.23 \pm 0.01^{\mathrm{a}}$ \\
\hline Acidez titulable (\%) & $0.03 \pm 0.01^{\mathrm{a}}$ & $0.03 \pm 0.01^{\mathrm{a}}$ & $0.03 \pm 0.01^{\mathrm{a}}$ & $0.03 \pm 0.01^{\mathrm{a}}$ & $0.03 \pm 0.01^{\mathrm{a}}$ & $0.03 \pm 0.01^{\mathrm{a}}$ \\
\hline Sólidos totales (\%) & $2.32 \pm 0.02^{b}$ & $2.35 \pm 0.01^{b}$ & $2.41 \pm 0.01^{\mathrm{a}}$ & $2.37 \pm 0.02^{\mathrm{a}}$ & $2.31 \pm 0.02^{b}$ & $2.37 \pm 0.02^{\mathrm{a}}$ \\
\hline $\begin{array}{l}\text { Fenoles totales } \\
\text { (mg EAG/L) }\end{array}$ & $101.49 \pm 0.3^{b}$ & $95.23 \pm 1.63^{c}$ & $116.05 \pm 0.31^{\mathrm{a}}$ & $101.36 \pm 1.75^{b}$ & $99.25 \pm 1.38^{b}$ & $128.29 \pm 4.12^{\mathrm{a}}$ \\
\hline $\begin{array}{l}\text { Flavonoides totales } \\
(\mathrm{mg} \mathrm{EC/L)}\end{array}$ & $43.41 \pm 4.33^{c}$ & $61.75 \pm 2.20^{\mathrm{b}}$ & $72.30 \pm 5.35^{\mathrm{a}}$ & $53.13 \pm 4.11^{b}$ & $64.80 \pm 1.73^{\mathrm{a}}$ & $70.36 \pm 3.36^{\mathrm{a}}$ \\
\hline $\begin{array}{l}\text { Taninos condensados } \\
\text { (mg EC/L) }\end{array}$ & ND & ND & ND & ND & ND & ND \\
\hline $\begin{array}{l}\text { Antocianinas totales } \\
(\mathrm{mg} \mathrm{EC3G/L)}\end{array}$ & $1.03 \pm 0.07^{\mathrm{a}}$ & $0.97 \pm 0.07^{a}$ & $0.89 \pm 0.14^{\mathrm{a}}$ & $1.09 \pm 0.09^{\mathrm{a}}$ & $1.11 \pm 0.10^{\mathrm{a}}$ & $1.20 \pm 0.14^{\mathrm{a}}$ \\
\hline $\begin{array}{l}\text { Ácido clorogénico } \\
\text { (mg/L) }\end{array}$ & $13.51 \pm 0.64^{b}$ & $20.29 \pm 1.25^{\mathrm{a}}$ & $\mathrm{ND}^{c}$ & $37.14 \pm 3.77^{\mathrm{a}}$ & $39.58 \pm 0.99^{a}$ & $N D^{b}$ \\
\hline $\begin{array}{l}\text { Ácido cafeico } \\
\text { (mg/L) }\end{array}$ & $\mathrm{ND}^{\mathrm{b}}$ & $N D^{b}$ & $16.46 \pm 0.96^{\mathrm{a}}$ & $\mathrm{ND}^{\mathrm{b}}$ & $\mathrm{ND}^{\mathrm{b}}$ & $33.26 \pm 0.44^{\mathrm{a}}$ \\
\hline $\begin{array}{l}\text { Ácido ferúlico } \\
\text { (mg/L) }\end{array}$ & $45.77 \pm 1.07^{b}$ & $48.18 \pm 0.99^{a b}$ & $48.90 \pm 0.41^{\mathrm{a}}$ & $36.46 \pm 0.55^{b}$ & $41.72 \pm 1.01^{\mathrm{a}}$ & $40.57 \pm 0.68^{\mathrm{a}}$ \\
\hline $\begin{array}{l}\text { DPPH } \\
(\mu \mathrm{mol} \text { ET/L) }\end{array}$ & $163.67 \pm 6.01^{b}$ & $176.44 \pm 6.31^{b}$ & $267.56 \pm 5.09^{\mathrm{a}}$ & $312.00 \pm 11.67^{b}$ & $311.44 \pm 5.09^{b}$ & $384.78 \pm 6.31^{\mathrm{a}}$ \\
\hline $\begin{array}{l}\text { ABTS } \\
(\mu \mathrm{mol} E T / L)\end{array}$ & $473.84 \pm 26.68^{a}$ & $461.24 \pm 36.43^{\mathrm{ab}}$ & $389.90 \pm 28.26^{b}$ & $519.30 \pm 5.84^{\mathrm{a}}$ & $527.90 \pm 38.21^{\mathrm{a}}$ & $449.30 \pm 12.34^{b}$ \\
\hline $\begin{array}{l}\text { FRAP } \\
(\mu \mathrm{mol} E T / L)\end{array}$ & $815.00 \pm 25.00^{c}$ & $921.67 \pm 7.64^{b}$ & $1283.33 \pm 55.75^{\mathrm{a}}$ & $1093.33 \pm 85.20^{b}$ & $1168.33 \pm 12.58^{b}$ & $1566.67 \pm 80.36^{a}$ \\
\hline
\end{tabular}

Diferente letra en la misma línea dentro de cada tipo de bebida de maíz indica diferencia estadística ( $\mathrm{P} \leq 0.05)$. Media \pm Desviación estándar $(\mathrm{n}=5) . \mathrm{ND}=$ no detectado

Different letters in same line of each kind of maize beverages indicate statistical differences ( $P \leq 0.05)$. Means \pm Standard deviation ( $n=5)$. ND $=$ not detected. 
de los sólidos al realizar la toma de muestra. Por otro lado, en la bebida de maíz morado los valores de $\mathrm{pH}$, sólidos totales y acidez titulable, aunque mostraron diferentes valores, estos no mostraron diferencia estadística en sus valores, por lo que podemos mencionar que los tratamientos de termosonicación y pasteurización no ocasionaron alteraciones considerables en los parámetros fisicoquímicos en las bebidas (Rabie et al., 2015: Jiménez-Sánchez et al., 2017; Rodríguez-Salinas et al., 2019).

\section{Compuestos fenólicos}

El contenido de fenoles totales de las bebidas mostró valores de 95.23 a 116.05 y 99.25 a 128.29 mg EAG/L en maíz blanco y morado, respectivamente (Tabla 3). En ambas bebidas el efecto de los tratamientos fue similar, se encontraron diferencias entre los tratamientos de cada tipo de bebida, los menores valores se encontraron en los tratamientos de termosonicación y los mayores en la pasteurización. Estos resultados se atribuyen a que la alta temperatura generada por el tratamiento de pasteurización puede provocar la hidrólisis del enlace $O$-glucosídico de los azúcares presentes, como en este la sacarosa añadida en la formulación, la cual después de un proceso de hidrolisis por tratamiento térmico del azúcar puede dejar disponible el grupo reductor de la glucosa y fructosa para reaccionar con un grupo amino y generar la llamada reacción de Maillard, generando compuestos como las melanoidinas, moléculas con capacidad antioxidante (Tamanna y Mahmood, 2015; Mondaca-Navarro et al., 2017). Además, el calentamiento también puede favorecer la hidrólisis de compuestos fenólicos presentes de forma ligada, debido a que en ambas bebidas se observó el mismo efecto en la pasteurización (Wang et al., 2014).

El contenido de flavonoides de las bebidas de maíz blanco y morado no mostraron diferencia, los valores encontrados fueron de 43.41 a 72.30 y 53.13 a 70.36 mg EC/L, respectivamente (Tabla 3). Los tratamientos de las bebidas mostraron diferencia, con los menores valores en el control y los mayores en pasteurización. Principalmente, el incremento en la identificación de flavonoides en las bebidas se puede atribuir a que en el maíz existen una amplia diversidad de grupos flavonoides como flavonoles (quercetina, rutina y ramnetina) y flavonas (luteolina) (Biesaga, 2011). Los flavonoides tienen una estructura básica conformada por dos anillos aromáticos ( $\mathrm{A}$ y $\mathrm{B}$ ) unidos por un puente de tres carbonos, donde se forma un anillo pirano (C) (De Souza y De Giovani, 2005) y la hidroxilación y/o metilación del anillo C origina los diferentes grupos flavonoides (Martínez-Flórez et al., 2002). Asimismo, los flavonoides son muy efectivos para formar complejos con iones metálicos, los cuales proporcionan diferentes colores a las flores (Santos et al., 2002; Amat et al., 2010). Por tanto, los flavonoides totales de una muestra se cuantifican con la adición de iones metálicos, como un por ejemplo de esto es el cloruro de aluminio $\left(\mathrm{AlCl}_{3}\right)$ que se utiliza para formar complejos de flavonoides con aluminio (Denni y Mammen, 2012). Sin embargo, una de las deficiencias del ensayo con $\mathrm{AlCl}_{3}$, es que interferencia de los alguno de los grupos hidroxilo por la formación de enlaces con azúcares (glicosídicos) en cualquiera de los carbonos de las posiciones $3,5,3^{\prime}$ o 4' de los anillos $B$ o $C$, respectivamente, evita la formación de complejos (flavonoide-metal) con el Aluminio (Al) (Denni y Mammen, 2012; Pękal y Pyrzynska, 2014).

Los flavonoles y flavonas, poseen la característica de generar enlaces glicosídicos en la posición 3 del anillo B del flavonoide (Martínez-Flórez et al., 2002), característica utilizada para mejorar su estabilidad frente a la radiación UV y proteger de la oxidación a los tejidos vegetales (Smith et al., 2000). Por tanto, en el presente experimento, el análisis de la bebida de maíz sin pasteurizar pudo provocar una subestimación de los flavonoides totales, debido al bloqueo existente en el enlace glicosídico característico de los flavonoles y flavonas del maíz, los cuales pueden evitar la formación del complejo metálico con el Al. Sin embargo, los flavonoides totales de la bebida fueron mayormente detectados después del proceso de pasteurización. Esta detección se puede atribuir a que el calentamiento extremo generado por la pasteurización puede generar hidrolisis en los enlaces glicosídicos, lo que permite a los flavonoides desbloquear sus grupos hidroxilo para formar complejos metálicos e incrementar su detección por métodos espectrofotométricos (Pękal y Pyrzynska, 2014; Rodríguez-Salinas et al., 2019). Lo anteriormente mencionado, sugiere que el incremento en la detección de flavonoides de las bebidas de maíz blanco y morado se debió principalmente al efecto del proceso de pasteurización, lo que podría eliminar la posibilidad de que el incremento en la detección de flavonoides totales sea atribuido al ligero aumento de sólidos totales, observado sólo en la bebida de maíz blanco, después de la pasteurización.

Los taninos condensados no fueron detectados en ninguna de las bebidas de maíz, lo que se atribuye principalmente a la poca cantidad de la harina de maíz presente en la formulación. Con respecto al contenido de antocianinas en las bebidas de maíz blanco y morado (Tabla 3), los resultados de este experimento mostraron diferencia significativa en el tipo bebida, obteniendo valores de 0.89 a 1.03 y 1.09 a $1.20 \mathrm{mg} \mathrm{EC3G/L}$ respectivamente, con los mayores valores en la bebida de maíz morado. En cuanto a los tratamientos de termosonicación y pasteurización aplicados a cada tipo de bebida no se observaron diferencias estadísticas en el contenido de antocianinas. Las bajas concentraciones de antocianinas se atribuyeron a la dilución de la harina de maíz en la bebida, además a que este tipo de compuestos son solubles en agua (Lao y Giusti, 2016), por lo que se requiere de un método eficiente para eliminar agua y concentrar las antocianinas.

El contenido de ácido clorogénico en las bebidas de maíz blanco y morado mostraron diferencia significativa, los valores encontrados fueron de 13.51 a 20.29 y 37.14 y 39.58 $\mathrm{mg} / \mathrm{L}$, respectivamente (Tabla 3), siendo la bebida de maíz morado la que mostró el mayor contenido de este ácido. En los tratamientos de la bebida de maíz blanco se encontró diferencia con el mayor contenido en el tratamiento de termosonicación (Figura 1, a y b). En la bebida de maíz morado 


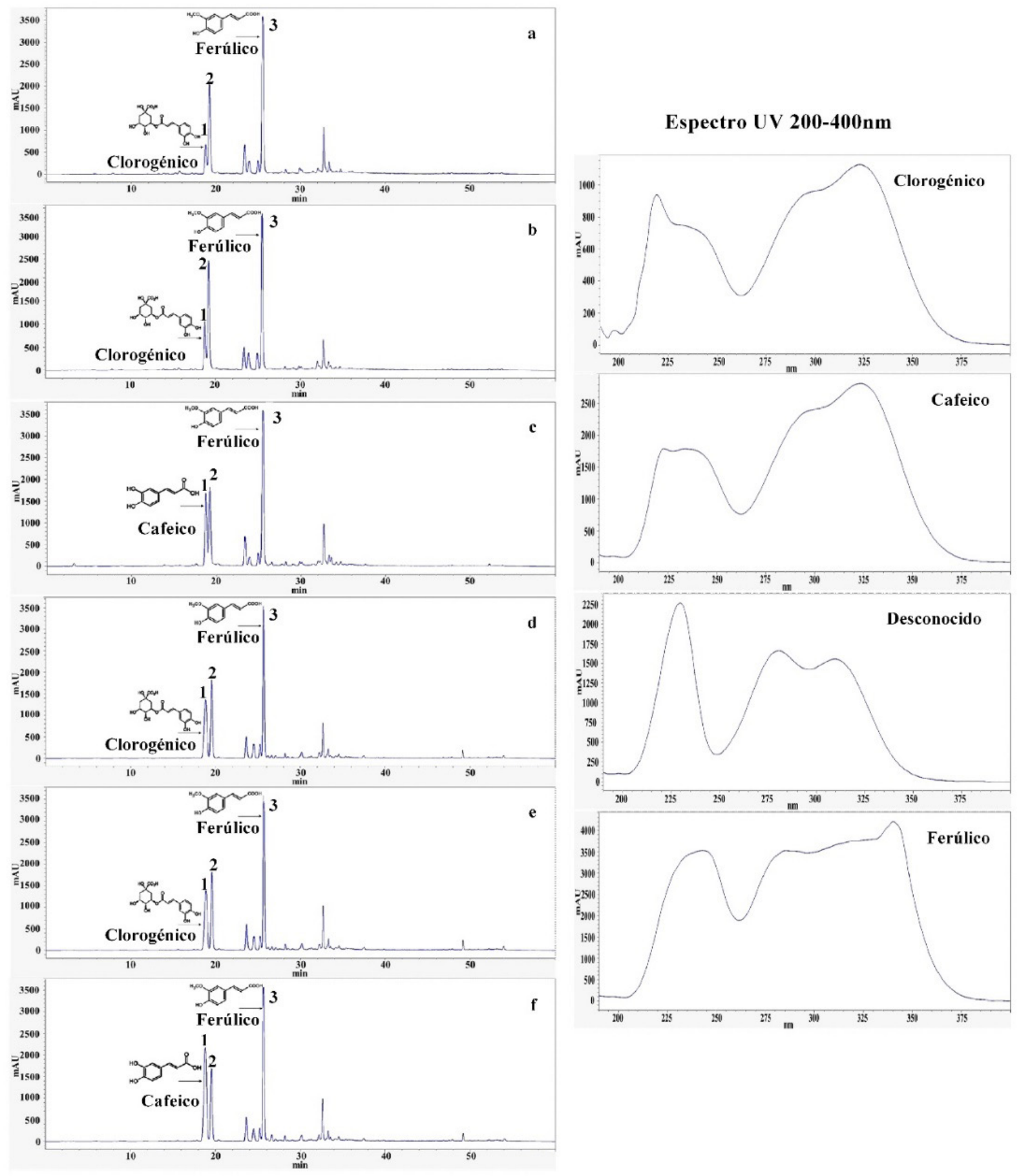

Figura 1. Cromatogramas de bebidas de maíz y espectro de UV-200-400 nm de los picos detectados.Bebida maíz blanco control, 1. Ácido clorogénico, 2. Desconocido, 3. Ácido ferúlico. Bebida maíz blanco con termosonicación, 1. Ácido clorogénico, 2. Desconocido, 3. Ácido ferúlico.

Bebida maíz blanco con pasteurización, 1. Ácido cafeico, 2. Desconocido, 3. Ácido ferúlico.

Bebida maíz morado control, 1. Ácido clorogénico, 2. Desconocido, 3. Ácido ferúlico.

Bebida maíz morado con termosonicación, 1. Ácido clorogénico, 2. Desconocido, 3. Ácido ferúlico.

Bebida maíz morado con pasteurización, 1. Ácido cafeico, 2. Desconocido, 3. Ácido ferúlico.

Figure 1. Chromatograms of maize beverages and UV spectrum 200-400 nm of peaks detected.

a) White maize beverage control, 1. Chlorogenic acid, 2. Unknown, 3. Ferulic acid.b) White thermosonicated maize beverage, 1. Chlorogenic acid, 2. Unknown, 3. Ferulic acid.c) White pasteurized maize beverage, 1. Caffeic acid, 2. Unknown, 3. Ferulic acid.d) Purple control maize beverage, 1. Chlorogenic acid, 2. Unknown, 3. Ferulic acid.e) Purple thermosonicated maize beverage, 1. Chlorogenic acid, 2. Unknown, 3. Ferulic acid.f) Purple pasteurized maize beverage, 1. Caffeic acid, 2. Unknown, 3. Ferulic acid. 
no se observó diferencia entre el control y termosonicación (Figura 1, d y e). En el tratamiento de pasteurización para cada una de las bebidas no se detectó el ácido clorogénico, sin embargo, sólo en estos tratamientos se detectó ácido cafeico (Figura 1, c y f), con valores de 16.46 y $33.26 \mathrm{mg} / \mathrm{L}$ en la bebida de maíz blanco y morado, respectivamente, con los valores más altos en la bebida de maíz morado.

Los resultados obtenidos mostraron que la termosonicación incrementó la detección y/o liberación de ácido clorogénico, ya que este puede encontrarse esterificado a los polisacáridos de la pared celular (Parr y Bolwell, 2000). Además, se observó que la termosonicación no mostró efecto de hidrólisis en la molécula del ácido clorogénico a diferencia de la pasteurización. En el tratamiento de pasteurización el ácido clorogénico no se detectó en ninguna de las bebidas, esto se debe a que la molécula de ácido clorogénico está constituida por un ácido quínico esterificado al ácido cafeico (Santos et al., 2014), y este enlace éster puede hidrolizarse por efecto térmico en soluciones acuosas (Rodríguez-Salinas et al., 2019).

Los valores de ácido ferúlico (Tabla 3), obtenidos de los cromatogramas de las bebidas de maíz blanco y morado (Figura 1, a y f), mostraron diferencias significativas en el tipo de bebida con valores de 45.77 a 48.90 y 36.46 a 41.72 mg/L, respectivamente, siendo la bebida de maíz blanco la que mostró el mayor contenido. En los tratamientos de ambas bebidas se observaron diferencias significativas con los menores valores en el control, mientras que los mayores valores en los tratamientos de termosonicación y pasteurización.

Los resultados en el contenido de ácido ferúlico mostraron un incrementó por efecto de los tratamientos de termosonicación y pasteurización, esto se atribuye a que ambos tratamientos tuvieron efecto de liberación de ácido ferúlico, que en los cereales es uno de los principales ácidos fenólicos encontrados de forma ligada a polisacáridos de la pared celular (Boz, 2015; Jiménez-Sánchez et al., 2017; Rodríguez-Salinas et al., 2019).

\section{Capacidad antioxidante}

La capacidad antioxidante en las bebidas de maíz blanco y morado mostraron diferencia significativa independientemente del método utilizado. Para DPPH se observaron valores de 163.67 a 267.56 y 311.48 a 384.78 umol ET/L, mientras que para el método de FRAP la capacidad antioxidante varió de 815.00 a 1283.33 y 1093.33 a $1566.67 \mu \mathrm{mol}$ ET/L, respetivamente, siendo la bebida de maíz morado la que mostró mayor capacidad antioxidante (Tabla 3). En todas las bebidas de maíz en estudio se observó el mismo comportamiento, la diferencia entre sus tratamientos fue del menor valor para el control y termosonicación, y el mayor en la pasteurización.

Además, es importante mencionar que para los métodos de DPPH y FRAP, los valores de capacidad antioxidante mostraron incrementos con la termosonicación y pasteurización. Durante la termosonicación ocurre cavitación, a lo que podría atribuirse el incremento del ácido ferúlico, el cual pudiera haber sido liberado de la pared celular (Abdullah y
Chin, 2014; Jiménez-Sánchez et al., 2017). Asimismo, para el tratamiento de pasteurización, la reacción de Maillard podría causar sinergismo entre los fitoquímicos y las melanoidinas, así como la liberación de ácido ferúlico, considerado como un potente antioxidante (Boz, 2015; Rodríguez-Salinas et al., 2019).

Para el método de ABTS, la capacidad antioxidante de las bebidas mostró diferencia significativa con valores que oscilaron de 389.90 a 473.84 y 449.30 a $527.90 \mu \mathrm{mol}$ ET/L en la de maíz blanco y morado, respectivamente, con la mayor capacidad antioxidante en la bebida de maíz morado (Tabla 3). En los tratamientos de las bebidas de maíz blanco y morado se encontró que el menor valor fue para la pasteurización, mientras que los mayores en control y termosonicación.

Asimismo, se pudo observar que la capacidad antioxidante en ABTS para ambas bebidas de maíz no se vieron afectadas por el tratamiento con temosonicación. Este efecto pudiera ser atribuido a que este tratamiento evita la degradación de compuestos sensibles al calor, y por lo tanto mantiene las características antioxidantes. Además, existe evidencia que el uso de la termosonicación inactiva enzimas causantes de la disminución de compuestos fenólicos, como es la polifenol oxidasa (Abdullah y Chin, 2014; JiménezSánchez et al., 2017). Sin embargo, la capacidad antioxidante en ABTS mostró disminución por efecto de la pasteurización, esto debido a que los procesos térmicos con alta temperatura posiblemente pueden provocar transformaciones químicas de compuestos fenólicos o formación de complejos de estos compuestos con otras sustancias (Salas et al., 2018) lo que impide su capacidad de ceder electrones (Rodríguez-Salinas et al., 2019).

\section{Viscosidad}

Los valores de viscosidad en las bebidas de maíz blanco y morado mostraron un comportamiento pseudoplástico, de acuerdo con la ley de potencia. No se observaron diferencias significativas entre las diferentes bebidas para el coeficiente de consistencia (K), y para el índice de flujo (n) (Tabla 4), tampoco se observaron diferencias significativas entre los tratamientos aplicados a cada tipo de bebida.

Los resultados en este trabajo mostraron que la viscosidad de las bebidas no mostró un efecto por el tratamiento de termosonicación. Los resultados obtenidos en este estudio coinciden con lo reportado por Anaya-Esparza et al. (2017), quienes mencionaron que el tratamiento con termosonicación no afecta la viscosidad, y además, podría mejorar la viscosidad, ya que favorece la distribución y disminuye el tamaño de los hidrocoloides.

Por otro lado, algunos estudios realizados con termosonicación han reportado pérdida de viscosidad, esto ocasionado por el daño permanente a los hidrocoloides, sin embargo, estos resultados dependen en gran medida a la energía y tiempo de aplicación del ultrasonido (Rojas y Augusto, 2018). En cuanto al tratamiento de pasteurización, este tampoco disminuyó la viscosidad, estos resultados corroboran las propiedades de las gomas como estabilizantes 
Tabla 4. Parámetros de viscosidad en bebidas de maíz blanco y maíz morado.

Table 4. Viscosity parameters in white and purple maize beverages.

\begin{tabular}{lcccccc}
\hline \multirow{2}{*}{$\begin{array}{l}\text { Parámetros de } \\
\text { viscosidad }\end{array}$} & \multicolumn{3}{c}{ Bebida de Maíz Blanco } & \multicolumn{3}{c}{ Bebida de maíz Morado } \\
\cline { 2 - 7 } & Control & Termosonicación & Pasteurización & Control & Termosonicación & Pasteurización \\
\hline K (Pas) & $-2.35 \pm 0.47^{\mathrm{a}}$ & $-3.24 \pm 0.76^{\mathrm{a}}$ & $-1.99 \pm 0.25^{\mathrm{a}}$ & $-2.52 \pm 0.44^{\mathrm{a}}$ & $-3.84 \pm 1.82^{\mathrm{a}}$ & $-2.29 \pm 0.18^{\mathrm{a}}$ \\
$\mathrm{N}$ & $0.63 \pm 0.09^{\mathrm{a}}$ & $0.76 \pm 0.14^{\mathrm{a}}$ & $0.58 \pm 0.04^{\mathrm{a}}$ & $0.65 \pm 0.08^{\mathrm{a}}$ & $0.83 \pm 0.33^{\mathrm{a}}$ & $0.64 \pm 0.03^{\mathrm{a}}$ \\
\hline
\end{tabular}

Diferente letra en la misma línea dentro de cada tipo de bebida de maíz indica diferencia estadística significativa $(P \leq 0.05)$. Media \pm Desviación estándar $(n=5)$.

Different letters in same line of each kind of maize beverages indicate significant statistical differences $(P \leq 0.05)$. Means \pm standard deviation $(n=5)$.

de bebidas y su uso efectivo frente a tratamientos térmicos (Paquet et al., 2014; Sun-Waterhouse et al., 2014).

\section{CONCLUSIONES}

Los tratamientos aplicados a las bebidas en estudio mostraron ligeros cambios en el color. Ambos tratamientos disminuyeron el conteo de microorganismos, sin embargo, a diferencia de la termosonicación, la pasteurización mostró el $100 \%$ de letalidad. Por tanto, es necesario evaluar el efecto del incremento de la fuerza del ultrasonido y/o combinarlo con otros métodos de tecnología emergente, para incrementar su eficiencia. Los dos tratamientos no afectaron la viscosidad, calidad fisicoquímica de las bebidas, y facilitaron la identificación y/o liberación de compuestos fenólicos.

Por otro lado, la pasteurización, provocó la hidrólisis de ácido clorogénico lo que incrementó la identificación del ácido cafeico, así como liberación del ácido ferúlico, lo que ocasionó un incremento de la capacidad antioxidante por los métodos de DPPH y FRAP en ambas bebidas. En ambas bebidas el contenido de compuestos fenólicos totales fue similar. En la bebida de maíz blanco se observó el mayor contenido de ácido ferúlico, mientras que en la de maíz morado destacaron el ácido clorogénico, cafeico y capacidad antioxidante. Por lo que se puede mencionar que la bebida de maíz morado mostró mayor capacidad antioxidante en comparación con la bebida de maíz blanco.

\section{AGRADECIMIENTOS}

Agradecemos el apoyo al programa del Consejo $\mathrm{Na}$ cional de Ciencia y Tecnología (CONACYT), a la Facultad de Agronomía, a la Facultad de Ciencias Biológicas de Universidad Autónoma de Nuevo León, al Centro de Investigación y Asistencia en Tecnología y Diseño del Estado de Jalisco, A.C., Sede Noreste, al Centro de Investigación en Alimentación y Desarrollo, A.C., Unidad Culiacán.

\section{REFERENCIAS}

Aamir, M., Ovissipour, M., Rasco, B., Tang, J., y Sablani, S. (2014). Seasonality of the thermal kinetics of color changes in whole spinach (Spinacia oleracea) leaves under pasteurization conditions. International Journal of Food Properties, 17(9): 2012-2024.

Abdullah, N., y Chin, N.L. (2014). Application of thermosonication treatment in processing and production of high quality and safe-to-drink fruit juices. Agriculture and Agricultural Science Procedia, 2: 320-327.

Akonor,P.T.,Tortoe, C.,yOduro-Yeboah, C. (2014).Physicochemical characterization of non-alcoholic beverages produced from malted roasted varieties of maize (Zea mays). International Journal of Food Science and Nutrition Engineering, 4(1): 20-26.

Amat, A., Clementi, C., Miliani, C., Romani, A., Sgamellotti, A., y Fantacci, S. (2010). Complexation of apigenin and luteolin in weld lake: a DFT/TDDFT investigation. Physical Chemistry Chemical Physics, 12(25): 6672-6684.

Anaya-Esparza, L.M., Velázquez-Estrada, R.M., Roig, A.X., GarcíaGalindo, H.S., Sayago-Ayerdi, S.G., y Montalvo-González, E. (2017). Thermosonication: An alternative processing for fruit and vegetable juices. Trends in Food Science \& Technology, 61:26-37.

Biesaga, M. (2011). Influence of extraction methods on stability of flavonoids. Journal of chromatography A, 1218(18): 25052512.

Boz, H. (2015). p-Coumaric acid in cereals: presence, antioxidant and antimicrobial effects. International Journal of Food Science \& Technology, 50(11): 2323-2328.

Carrera, Y., Utrilla-Coello, R., Bello-Pérez, A., Alvarez-Ramirez, J., y Vernon-Carter, E.J. (2015). In vitro digestibility, crystallinity, rheological, thermal, particle size and morphological characteristics of pinole, a traditional energy food obtained from toasted ground maize. Carbohydrate Polymers, 123: 246-255.

Chaves-López, C., Serio, A., Grande-Tovar, C.D., Cuervo-Mulet, R., Delgado-Ospina, J., y Paparella, A. (2014). Traditional fermented foods and beverages from a microbiological and nutritional perspective: the Colombian heritage. Comprehensive Reviews in Food Science and Food Safety, 13(5): 1031-1048.

De Souza, R.F., y De Giovani, W.F. (2005). Synthesis, spectral and electrochemical properties of $\mathrm{Al}$ (III) and $\mathrm{Zn}$ (II) complexes with flavonoids. Spectrochimica Acta Part A: Molecular and Biomolecular Spectroscopy, 61(9): 1985-1990.

Denni, M., y Mammen, D. (2012). A critical evaluation on the reliability of two aluminum chloride chelation methods for quantification of flavonoids. Food chemistry, 135(3): 13651368.

Estrada, E., Lengua, R., Pino, J., Alvis, R., Bazán, D., Becerra, E., Sandívar, J., Carhuacho, M., Osorio, A., y Caja, V.R. (2010). Proceso para obtener bebida nutracéutica a partir de Myrciaria Dubia (Camu Camu), orientado a reducir efecto genotóxico en niños de edad escolar. Revista Peruana de Química e Ingeniería Química, 12(2): 34-41.

Jiménez-Sánchez, C., Lozano-Sánchez, J., Segura-Carretero, A., y Fernández-Gutiérrez, A. (2017). Alternatives to conventional 
thermal treatments in fruit-juice processing. Part 2: Effect on composition, phytochemical content, and physicochemical, rheological, and organoleptic properties of fruit juices. Critical Reviews in Food Science and Nutrition, 57(3): 637652.

Kathiravan, T., Nadanasabapathi, S., y Kumar, R. (2014). Standardization of process condition in batch thermal pasteurization and its effect on antioxidant, pigment and microbial inactivation of Ready to Drink (RTD) beetroot (Beta vulgaris L.) juice. International Food Research Journal, 21(4): 1305-1312.

Lao, F., y Giusti, M.M. (2016). Quantification of purple corn (Zea mays L.) anthocyanins using spectrophotometric and HPLC approaches: method comparison and correlation. Food Analytical Methods, 9(5): 1367-1380.

López-Contreras, J.J., Zavala-Garcia, F., Urias-Orona, V., MartinezAvila, G.C.G., Rojas, R., y Guillermo, N.M. (2015). Chromatic, phenolic and antioxidant properties of Sorghum bicolor genotypes. Notulae Botanicae Horti Agrobotanici ClujNapoca, 43(2): 366-370.

Martínez-Flórez, S., González-Gallego, J., Culebras, J.M., y Tuñón, M. (2002). Los flavonoides: propiedades y acciones antioxidantes. Nutrición hospitalaria, 17(6): 271-278.

Mondaca-Navarro, B.A., Ávila-Villa, L.A., González-Córdova, A.F., López-Cervantes, J., Sánchez-Machado, D.I., CampasBaypoli, O.N., y Rodríguez-Ramírez, R. (2017). Antioxidant and chelating capacity of Maillard reaction products in amino acid-sugar model systems: applications for food processing. Journal of the Science of Food and Agriculture, 97(11): 3522-3529.

Niño-Medina, G., Muy-Rangel, D., y Urías-Orona, V. (2017). Chickpea (Cicer arietinum) and soybean (Glycine max) hulls: byproducts with potential use as a source of high valueadded food products. Waste and Biomass Valorization, 8(4): 1199-1203.

Paquet, É., Hussain, R., Bazinet, L., Makhlouf, J., Lemieux, S., y Turgeon, S.L. (2014). Effect of processing treatments and storage conditions on stability of fruit juice based beverages enriched with dietary fibers alone and in mixture with xanthan gum. LWT-Food Science and Technology, 55(1): 131-138.

Parr, A.J., y Bolwell, G.P. (2000). Phenols in the plant and in man. The potential for possible nutritional enhancement of the diet by modifying the phenols content or profile. Journal of the Science of Food and Agriculture, 80(7): 985-1012.

Pękal, A., y Pyrzynska, K. (2014). Evaluation of aluminium complexation reaction for flavonoid content assay. Food Analytical Methods, 7(9): 1776-1782.

Rabie, M.A., Soliman, A.Z., Diaconeasa, Z.S., y Constantin, B. (2015). Effect of Pasteurization and Shelf Life on the Physicochemical Properties of Physalis (Physalis peruviana L.) Juice. Journal of Food Processing and Preservation, 39(6): 1051-1060.

Ranum, P., Peña-Rosas, J.P., Garcia-Casal, y M.N. (2014). Global maize production, utilization, and consumption. Annals of the New York Academy of Sciences, 1312(1): 105-112.

Rawson, A., Patras, A., Tiwari, B.K., Noci, F., Koutchma, T., y Brunton, N. (2011). Effect of thermal and non thermal processing technologies on the bioactive content of exotic fruits and their products: Review of recent advances. Food Research International, 44(7): 1875-1887.

Rico, D., Martin-Diana, A.B., Barat, J.M., y Barry-Ryan, C. (2007). Extending and measuring the quality of fresh-cut fruit and vegetables: a review. Trends in Food Science \& Technology, 18(7): 373-386.

Rodríguez-Salinas, P.A., Muy-Rangel, D., Urias-Orona, V., ZavalaGarcia, F., Suarez-Jacobo, A., Heredia, J.B., Rubio-Carrasco, W., y Nino-Medina, G. (2019). Thermal processing effects on the microbiological, physicochemical, mineral, and nutraceutical properties of a roasted purple maize beverage. Farmacia, 67(4): 587-595.

Rojas, M.L., y Augusto, P.E. (2018). Ethanol and ultrasound pre-treatments to improve infrared drying of potato slices. Innovative Food Science \& Emerging Technologies, 49: 6575.

Roig-Sagués, A.X., Asto, E., Engers, I., y Hernández-Herrero, M.M. (2015). Improving the efficiency of ultra-high pressure homogenization treatments to inactivate spores of Alicyclobacillus spp. in orange juice controlling the inlet temperature. LWT-Food Science and Technology, 63(2): 866871.

Salas, R., Ordoñez, E., y Reátegui, D. (2018). Polifenoles totales y capacidad antioxidante (DPPH y ABTS) en cuatro variedades de frejol (Phaseolus vulgaris L.) crudo seco, remojado y cocido. Revista Investigación y Amazonia, 5 (1 y 2): 55-62.

Santos, J.P., Zaniquelli, M.E.D., De Giovani, W.F., y Galembeck, S.E. (2002). Aluminum ion complex formation with 3-hydroxyflavone in Langmuir and Langmuir-Blodgett films. Colloids and Surfaces A: Physicochemical and Engineering Aspects, 198: 569-576.

Santos, J., Oliveira, M.B.P.P., Ibáñez, E., y Herrero, M. (2014). Phenolic profile evolution of different ready-to-eat babyleaf vegetables during storage. Journal of Chromatography A, 1327: 118-131.

Smith, G.J., Thomsen, S.J., Markham, K.R., Andary, C., y Cardon, D. (2000). The photostabilities of naturally occurring 5-hydroxyflavones, flavonols, their glycosides and their aluminium complexes. Journal of Photochemistry and Photobiology A: Chemistry, 136(1-2): 87-91.

Suárez-Jacobo, Á., Gervilla, R., Guamis, B., Roig-Sagués, A.X., y Saldo, J. (2010). Effect of UHPH on indigenous microbiota of apple juice: a preliminary study of microbial shelf-life. International journal of food microbiology, 136(3): 261-267.

Suárez-Jacobo, Á., Rüfer, C.E., Gervilla, R., Guamis, B., Roig-Sagués, A.X., y Saldo, J. (2011). Influence of ultra-high pressure homogenisation on antioxidant capacity, polyphenol and vitamin content of clear apple juice. Food chemistry, 127(2): 447-454.

Sun-Waterhouse, D., Bekkour, K., Wadhwa, S.S., y Waterhouse, G.I. (2014). Rheological and chemical characterization of smoothie beverages containing high concentrations of fibre and polyphenols from apple. Food and Bioprocess Technology, 7(2): 409-423.

Tamanna, N., y Mahmood, N. (2015). Food processing and maillard reaction products: effect on human health and nutrition. International journal of food science.

Urias-Lugo, D.A., Heredia, J.B., Serna-Saldivar, S.O., Muy-Rangel, M.D., y Valdez-Torres, J.B. (2015). Total phenolics, total anthocyanins and antioxidant capacity of native and elite blue maize hybrids (Zea mays L.). CyTA-Journal of Food, 13(3): 336-339.

Wang, T., He, F., y Chen, G. (2014). Improving bioaccessibility and bioavailability of phenolic compounds in cereal grains through processing technologies: A concise review. Journal of Functional Foods, 7: 101-111. 